J. Math. Biol. (1997) 36: 201-226

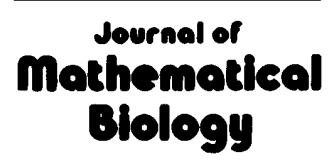

(C) Springer-Verlag 1997

\title{
The effect of periodic habitat fluctuations on a nonlinear insect population model
}

\author{
Shandelle M. Henson, J. M. Cushing* \\ Department of Mathematics, Building No. 89, Interdisciplinary Program on Applied \\ Mathematics, University of Arizona, Tucson, AZ 85721, USA
}

Received 23 April 1996; received in revised form 28 March 1997

\begin{abstract}
Laboratory data show that populations of flour beetles (Tribolium), when grown in a periodically fluctuating volume of flour, can exhibit significant increases in numbers above those attained when grown in a constant volume (of the same average). To analyze and explain this phenomenon a discrete stagestructured model of Tribolium dynamics with periodic environmental forcing is introduced and studied. This model is an appropriately modified version of an experimentally validated model for flour beetle populations growing in a constant volume of flour, in which cannibalism rates are assumed inversely proportional to flour volume. This modeling assumption has been confirmed by laboratory experiments. Theorems implying the existence and stability of periodic solutions of the periodically forced model are proved. The time averages of periodic solutions of the forced model are compared with the equilibrium levels of the unforced model (with the same average flour volume). Parameter constraints are determined for which the average population numbers in the periodic environment are greater than (or less than) the equilibrium population numbers in the associated constant environment. Sample parameter estimates taken from the literature show that these constraints are fulfilled. These theoretical results provide an explanation for the experimentally observed increase in flour beetle numbers as a result of periodically fluctuating flour volumes. More generally, these integrated theoretical and experimental results provide the first convincing example illustrating the possibility of increased population numbers in a periodically fluctuating environment.
\end{abstract}

\section{Introduction}

In the natural world a biological organism's physical environment is often, if not usually, nonconstant in time. Consequently, fluctuating environments are 
of particular interest to population biologists. Despite this fact, the vast majority of mathematical models used in population dynamics and ecology are autonomous and assume a constant environment. As a result, virtually all fundamental principles in theoretical population dynamics are based upon the assumption of a constant environment: monotonic logistic growth, competitive exclusion and ecological niche, predator-prey oscillations, and so on. In recent years, non-autonomous versions of classical model equations have been investigated in order to determine the extent to which these fundamental principles remain valid in fluctuating environments. Both stochastic model equations (modeling stochastic fluctuations of the environment, at one extreme) and periodically forced equations (modeling regular periodic fluctuations of the environment, at the other extreme) have been studied; e.g. see $[1-6,8-12,17-22,26,27,30,31,35,36,42,43,46,47,49-53,55]$ and the references cited therein.

One tenet that resulted from early investigations of nonautonomous differential equation models for the growth of a single population was that a fluctuating environment has a deleterious effect. This piece of biological folklore seems to have been based primarily on the investigation of nonautonomous versions of the famous logistic equation. In [43, 44, 48], for example, periodically and stochastically forced logistic models of the form

$$
x^{\prime}=r x\left(1-\frac{x}{K_{0}+\gamma(t)}\right)
$$

are considered where $\gamma(t)$ is periodic or white noise with mean zero. It is shown that ave $\langle x\rangle<K_{0}$, i.e. that the average population size in a fluctuating environment is less than the equilibrium population level $K_{0}$ in the associated constant environment. On the other hand, in $[49,22]$ it is shown that this conclusion is model dependent. For example, in [22] it is shown that if the inherent growth rate $r$ in the periodic logistic equation is also allowed to vary periodically, then ave $\langle x\rangle$ can be greater than the average carrying capacity $K_{0}$. Furthermore, in $[22,49,7]$ it is shown that properties of the nonlinearity play an important role (particularly, the concavity).

Thus, in theory, the average total biomass of a population in a periodic environment can be greater or less than the average total biomass in the associated constant average habitat, depending on the nonlinearities present and the specific properties of the forcing and inherent periodicities (such as, for example, their phase relationship). If the advent of environmental periodicity causes an increase in the average total population number of all individuals, we will say that the periodicity has a "positive effect". If a decrease in average occurs we will say that the periodicity has a "negative effect". (We will also speak, in a similar way, of positive and negative effects on the average numbers in individual life cycle stages as well.)

Are there any population data that provide evidence of a positive effect due to environmental periodicity? There are in fact little data, either field or laboratory, that specifically address the effect of periodically fluctuating 
environments on population density. Furthermore, there are preciously few rigorously validated mathematical models in population dynamics that could be used to describe and explain such data if it existed. Consequently, while a positive effect of environmental periodicity is a theoretical possibility, there are little replicated and controlled experimental data and few rigorously validated models available to support it. An exception comes from the controlled laboratory experiments utilizing flour beetles (Tribolium) conducted by Jillson [40] and the stage structured model that has been recently developed and tested for the population dynamics of flour beetles [14, 15, 25, 28, 29].

In his experiments Jillson placed flour beetles in volumes of flour that were alternated between 32 grams and 8 grams every two weeks. The control beetle populations were kept in a constant flour volume of 20 grams. Jillson found that total population numbers in the periodically fluctuating environment were more than twice those in the constant environment, even though the average flour volume was the same in both cases. This is a striking example of a positive effect of a periodically fluctuating environment.

The dynamical model for flour beetle dynamics used in [14, 15, 25, 28, 29] was developed and laboratory tested for a constant habitat (i.e. flour volume). This discrete, stage structured model, which is based upon the life cycle characteristics of flour beetles, has been thoroughly validated in [14, 28, 29] by means of controlled, replicated laboratory experiments and extensive statistical tests. We will show that a periodically forced modification of this model, derived from the assumption of a periodically varying volume of flour, can predict increased population numbers of beetles under certain circumstances and decreased numbers under other circumstances. Specifically, it will be shown that the model predicts a positive effect of environmental periodicity when the inherent larval recruitment rate is sufficiently large and the flour volume oscillation has small (relative) amplitude. It will also be shown that the model predicts a negative effect of environmental periodicity for small larval recruitment rates at any (relative) amplitude. The periodically forced modification of the autonomous model is based on a modeling assumption recently validated by controlled laboratory experiments [16]. This assumption is that the environmental fluctuations affect only the nonlinear interactions between life cycle stages (in this case cannibalism).

Our results here offer a specific explanation for the unusual increased biomass in a fluctuating environment observed in Jillson's flour beetle experiments, namely fluctuating cannibalism rates (which are inversely proportional to flour volume). A more detailed analysis of the actual Jillson experiment using the periodic model studied here will be given elsewhere [16]. These results provide the first rigorous evidence, via model analysis of laboratory data, that an environmental periodicity can have a positive effect on total population biomass. From a broader prospective, we can say these results provide another counterexample to the classical assertion that environmental periodicities result in a negative effect, but one that is based firmly on controlled and replicated laboratory experiments and a rigorously validated model for the organism involved. 
We begin in Sect. 2 by describing the extant autonomous model along with a few of its properties. In Sect. 3 the periodic version of the model is derived and some fundamental facts about its dynamics proved, including persistence with respect to the extinction state for sufficiently large larval recruitment rates. Two existence theorems for 2-cycle solutions of the periodic model are proved in Sect. 4 using bifurcation and perturbation methods. In Sect. 5 the average of the 2-cycle solutions will be compared to the equilibrium levels in the environment with the same average flour volume. These results are derived by means of perturbation methods.

The concluding Sect. 6 contains a short discussion of some experimental data sets for which the theory applies and predicts a positive effect due to environmental periodicity, at least for large larval recruitment rates and small amplitude oscillations. Numerical results show that for these estimated parameter values it is often (but not always) the case that the predicted positive effects in fact occur for small larval recruitment rates and large amplitude oscillations as well.

\section{The autonomous model}

Costantino et al. $[14,25,28]$ have constructed and statistically validated a discrete stage-structured model of laboratory populations of flour beetles:

$$
\begin{aligned}
& L(t+1)=b A(t) \exp \left(-c_{e l} L(t)-c_{e a} A(t)\right) \\
& P(t+1)=\left(1-\mu_{l}\right) L(t) \\
& A(t+1)=P(t) \exp \left(-c_{p a} A(t)\right)+\left(1-\mu_{a}\right) A(t)
\end{aligned}
$$

We will refer to this model as the "LPA model". $L(t)$ denotes the number of larvae (feeding larvae), $P(t)$ denotes the number of pupae (nonfeeding larvae, pupae, and callow adults), and $A(t)$ denotes the number of adults. The discrete time interval is two weeks. The coefficient $b>0$ denotes the average number of larvae recruited per adult per unit time in the absence of cannibalism, $\mu_{l} \in(0,1)$ and $\mu_{a} \in(0,1)$ are the larval and adult probabilities of dying from causes other than cannibalism, and the exponentials represent the probabilities that individuals survive cannibalism one unit of time, with "cannibalism coefficients" $c_{e l}, c_{e a}, c_{p a}>0$. This model is based on the assumption that the dominant mechanism driving flour beetle dynamics is that of cannibalism between life cycle stages [13].

We now mention a few facts about the autonomous LPA model. Define

$$
b_{c r} \doteq \frac{\mu_{a}}{1-\mu_{l}}
$$

Theorem 1 There exists a positive equilibrium $\left(L_{0}, P_{0}, A_{0}\right)$ of the LPA model (1) if and only if $b>b_{c r}$. When it exists, this equilibrium is unique and each component is an unbounded, monotonically increasing function of $b$. 
Proof. There exists a positive equilibrium of the LPA model (1) if and only if there exists a solution pair $L>0$ and $A>0$ of the two algebraic equations

$$
\begin{aligned}
& L=b A \exp \left(-c_{e l} L-c_{e a} A\right) \\
& A=\frac{\left(1-\mu_{l}\right)}{\mu_{a}} L \exp \left(-c_{p a} A\right) .
\end{aligned}
$$

By solving the second equation for $L$ and substituting the result into the first equation we find that such a solution pair exists if and only if the equation

$$
b=\frac{\mu_{a}}{\left(1-\mu_{l}\right)} \exp \left[A\left(c_{e a}+c_{p a}\right)\right] \exp \left[c_{e l} \frac{\mu_{a}}{\left(1-\mu_{l}\right)} A \mathrm{e}^{c_{p a} A}\right]
$$

has a positive solution $A=A_{0}>0$. Since the right hand side of equation (2) is an unbounded, monotonically increasing function of $A$ with value $b_{c r}$ at $A=0$, equation (2) has a positive solution $A_{0}$ if and only if $b>b_{c r}$, and this solution is unique when it exists. Moreover, $A_{0}$ is a strictly increasing, unbounded function of $b>b_{c r}$. From the solution $A_{0}$ of equation (2), the equations

$$
\begin{aligned}
L_{0} & =\frac{\mu_{a}}{\left(1-\mu_{l}\right)} A_{0} \exp \left(c_{p a} A_{0}\right) \\
P_{0} & =\left(1-\mu_{l}\right) L_{0}
\end{aligned}
$$

provide unique positive $L_{0}$ and $P_{0}$. Formula (3) shows $L_{0}$ and $P_{0}$ are also strictly increasing, unbounded functions of $b$.

Let $R_{+}^{3} \doteq[0, \infty) \times[0, \infty) \times[0, \infty)$. The following results are shown in [23].

Theorem 2 The trivial equilibrium $(L, P, A)=(0,0,0)$ of the LPA model (1) is globally asymptotically stable if $b<b_{c r}$ and unstable if $b>b_{c r}$. If $b>b_{c r}$, the solution in Theorem 1 is locally asymptotically stable for $b$ sufficiently close to $b_{c r}$. In any case, for all $b>b_{c r}$, the system (1) is uniformly persistent with respect to the extinction state $(0,0,0)$, i.e., there exists $\varepsilon>0$ such that $\liminf _{t \rightarrow \infty}\|(L(t), P(t), A(t))\|>\varepsilon \quad$ for all orbits with $\quad(L(0), P(0), A(0)) \in$ $R_{+}^{3}-\{(0,0,0)\}$.

For other results see [41].

\section{The periodically forced model}

A common protocol in flour beetle experiments is to replace the medium with fresh flour at each census with volumes sufficient to insure little if any food shortages and hence little if any intra-specific competition or density effects (e.g. see [28, 40]). Therefore, in our model it is assumed that only the cannibalism rates are affected by changes in flour volume. Cannibalism in Tribolium occurs during random encounters as larvae and adult beetles roam 
through their flour habitat. Therefore, cannibalism rates can reasonably be assumed to be inversely related to flour volume V [13, 54]. Recent laboratory experiments carried out by R. A. Desharnais [16] have shown, more specifically, that cannibalism rates are inversely proportional to flour volume. This fact can be accounted for in the LPA model (1) by assuming the cannibalism coefficients take the forms

$$
c_{e l}=\frac{\kappa_{e l}}{V}, \quad c_{e a}=\frac{\kappa_{e a}}{V}, \quad c_{p a}=\frac{\kappa_{p a}}{V} .
$$

If flour volume periodically oscillates with a period of 2 and an amplitude of $\alpha V_{\text {ave }}$ around an average $V_{\text {ave }}$ then $V=V_{\text {ave }}\left(1+\alpha(-1)^{t}\right)$ and the cannibalism coefficients in the LPA model become, respectively,

$$
\frac{\kappa_{e l}}{V_{\text {ave }}\left(1+\alpha(-1)^{t}\right)}, \quad \frac{\kappa_{e a}}{V_{\text {ave }}\left(1+\alpha(-1)^{t}\right)}, \quad \frac{\kappa_{p a}}{V_{\text {ave }}\left(1+\alpha(-1)^{t}\right)} .
$$

Let $c_{e l}, c_{e a}$, and $c_{p a}$ denote the cannibalism coefficients in the average volume of flour, i.e.

$$
c_{e l} \doteq \frac{\kappa_{e l}}{V_{a v e}}, \quad c_{e a} \doteq \frac{\kappa_{e a}}{V_{a v e}}, \quad c_{p a} \doteq \frac{\kappa_{p a}}{V_{a v e}} .
$$

Let $N$ denote the nonnegative integers. We then obtain the "periodic $L P A$ model"

$$
T: N \times R_{+}^{3} \rightarrow R_{+}^{3}
$$

which is described componentwise by the equations

$$
\begin{aligned}
& L(t+1)=b A(t) \exp \left(-\frac{c_{e l}}{1+\alpha(-1)^{t}} L(t)-\frac{c_{e a}}{1+\alpha(-1)^{t}} A(t)\right) \\
& P(t+1)=\left(1-\mu_{l}\right) L(t) \\
& A(t+1)=P(t) \exp \left(-\frac{c_{p a}}{1+\alpha(-1)^{t}} A(t)\right)+\left(1-\mu_{a}\right) A(t)
\end{aligned}
$$

for relative amplitude $\alpha \in[0,1)$. Note that the autonomous LPA model (1) is obtained by setting $\alpha=0$ in this periodic LPA model and by interpreting the cannibalism coefficients as those associated with a constant environment with volume of flour fixed at $V_{\text {ave }}$.

We now consider a few general mathematical facts about the periodic LPA model (4) before turning our attention to the existence, uniqueness, and stability of positive 2-cycle solutions. Define the autonomous maps

as the composites

$$
\begin{aligned}
& T_{1}: R_{+}^{3} \rightarrow R_{+}^{3} \\
& T_{2}: R_{+}^{3} \rightarrow R_{+}^{3}
\end{aligned}
$$

$$
\begin{aligned}
& \hat{x}(t+1)=T_{1}(\hat{x}(t)) \doteq T(1, T(0, \hat{x}(t))) \\
& \hat{y}(t+1)=T_{2}(\hat{y}(t)) \doteq T(2, T(1, \hat{y}(t))) .
\end{aligned}
$$


Then

whenever

$$
\begin{aligned}
& \hat{x}(t)=(L(2 t), P(2 t), A(2 t)) \\
& \hat{y}(t)=(L(2 t+1), P(2 t+1), A(2 t+1))
\end{aligned}
$$

$$
\begin{aligned}
& \hat{x}(0)=(L(0), P(0), A(0)) \\
& \hat{y}(0)=(L(1), P(1), A(1)) .
\end{aligned}
$$

Theorem 3 The trivial solution $\hat{0}=(0,0,0)$ of the periodic LPA model (4) is globally asymptotically stable if $b<b_{c r}$ and unstable if $b>b_{c r}$.

Proof. Let $b>b_{c r}$. The Jacobians of both $T_{1}$ and $T_{2}$ at 0 have characteristic equation

$$
\lambda^{3}-\left(1-\mu_{a}\right)^{2} \lambda^{2}-2 \lambda b\left(1-\mu_{a}\right)\left(1-\mu_{l}\right)-\left(1-\mu_{l}\right)^{2} b^{2}=0 .
$$

Since the sum of the coefficients is negative, that is

$$
\begin{gathered}
1-\left(1-\mu_{a}\right)^{2}-2 b\left(1-\mu_{a}\right)\left(1-\mu_{l}\right)-\left(1-\mu_{l}\right)^{2} b^{2} \\
<2 \mu_{a}-\mu_{a}^{2}-2 \mu_{a}\left(1-\mu_{a}\right)-\mu_{a}^{2}=0,
\end{gathered}
$$

at least one eigenvalue is outside the unit circle. Hence the trivial solutions of both $T_{1}$ and $T_{2}$ are unstable, and so the trivial solution of (4) is unstable.

Let $b<b_{c r}$. For all $t \geqq 0$,

$$
\begin{aligned}
& L(t+1) \leqq b A(t) \\
& P(t+1) \leqq\left(1-\mu_{l}\right) L(t) \\
& A(t+1) \leqq P(t)+\left(1-\mu_{a}\right) A(t) .
\end{aligned}
$$

Thus, by induction, the solutions of the periodic LPA model (4) are bounded above by the solutions of the linearization of the LPA model (1) at zero, which has characteristic equation

$$
\lambda^{3}+\left(\mu_{a}-1\right) \lambda^{2}+\left(\mu_{l}-1\right) b=0 .
$$

It is easy to show (using, for example, the Jury Conditions [45] or Theorem 3 in Cushing and Zhou [24]) that all the eigenvalues $\lambda$ are inside the unit circle if and only if $b<b_{c r}$. Thus, the solutions of model (4) are bounded above by sequences which monotonically decrease to $\hat{0}$, and so the trivial solution of model (4) is globally asymptotically stable.

In order to show the uniform persistence of system (4), we need the following lemmas.

Lemma $1 T, T_{1}$, and $T_{2}$ are point dissipative, i.e., for each of $T, T_{1}$, and $T_{2}$ there exists a bounded set into which every orbit eventually enters and remains.

Proof. Consider the periodic LPA model (4). Let

$$
D \doteq \frac{b(1+\alpha)}{c_{e a}} \text {. }
$$


Then for all $t \geqq 2$

$$
\begin{aligned}
& L(t+1) \leqq b A(t) \exp \left(-\frac{c_{e a} A(t)}{1+\alpha}\right) \leqq D \\
& P(t+1) \leqq\left(1-\mu_{l}\right) L(t) \leqq\left(1-\mu_{l}\right) D \leqq D \\
& A(t+1) \leqq P(t)+\left(1-\mu_{a}\right) A(t) \leqq D+\left(1-\mu_{a}\right) A(t)
\end{aligned}
$$

and so $L$ and $P$ are forward bounded. By induction, $A(t)$ is bounded above by the solution $x(t)$ of the one-dimensional map

$$
\begin{aligned}
x(t+1) & =D+\left(1-\mu_{a}\right) x(t) \\
x(2) & =A(2)
\end{aligned}
$$

for $t \geqq 2$. The solution of this map is given by

$$
\begin{aligned}
x(t) & =D\left(\frac{1-\left(1-\mu_{a}\right)^{t-2}}{\mu_{a}}\right)+\left(1-\mu_{a}\right)^{t-2} x(2) \\
& =\frac{\left(1-\mu_{a}\right)^{t-2}\left(\mu_{a} x(2)-D\right)+D}{\mu_{a}} .
\end{aligned}
$$

Since $1-\mu_{a}<1, x(t)$ is monotonically decreasing to $D / \mu_{a}$ if $x(2)>D / \mu_{a}$ and monotonically increasing to $D / \mu_{a}$ if $x(2)<D / \mu_{a}$.

All orbits $(L, P, A)$ of the periodic LPA model (4) therefore eventually enter and remain in the rectangle

$$
[0, D] \times[0, D] \times\left[0,2 D / \mu_{a}\right] .
$$

Thus, $T$ is point dissipative, and so clearly $T_{1}$ is point dissipative.

To show that $T_{2}$ is point dissipative, it is sufficient to note that the phase-shifted model given componentwise by

$$
\begin{aligned}
& x(t+1)=b z(t) \exp \left(\frac{-c_{e l} x(t)-c_{e a} z(t)}{1+\alpha(-1)^{t+1}}\right) \\
& y(t+1)=\left(1-\mu_{l}\right) x(t) \\
& z(t+1)=y(t) \exp \left(\frac{-c_{p a} z(t)}{1+\alpha(-1)^{t+1}}\right)+\left(1-\mu_{a}\right) z(t)
\end{aligned}
$$

is dissipative by the same argument as above.

Lemma 2 The models defined by $T_{1}$ and $T_{2}$ have global attractors, i.e., for each of $T_{1}$ and $T_{2}$, there is a maximal compact invariant set $A_{i}$ such that $\omega_{i}(U) \doteq \bigcap_{m \geqq 0} c l \bigcup_{n \geqq m} T_{i}^{n} U \subseteq A_{i}$ for every bounded set $U \subseteq R_{+}^{3}$.

Proof. Since $T_{1}$ and $T_{2}$ are continuous on $R_{+}^{3}$ and point dissipative (and hence orbits of bounded sets are bounded), $T_{1}$ and $T_{2}$ have global attractors [34]. 
Lemma 3 Let $b>b_{c r}$. There exists an $\varepsilon>0$ such that if $\hat{x}(t)$ is any solution of (5) or (6) with $0<\|\hat{x}(0)\|<\varepsilon$, then there is $a \tau \geqq 1$ for which $\|\hat{x}(\tau)\|>\varepsilon$.

Proof. The Jacobian $J^{2}$ of both the composites $T_{1}$ and $T_{2}$ is

$$
J^{2}=\left(\begin{array}{ccc}
0 & b & b\left(1-\mu_{a}\right) \\
0 & 0 & b\left(1-\mu_{l}\right) \\
1-\mu_{l} & 1-\mu_{a} & \left(1-\mu_{a}\right)^{2}
\end{array}\right) .
$$

Since $J^{4}>0, J^{2}$ is primitive and irreducible and thus has a strictly dominant eigenvalue $\lambda$ which is simple and positive. Its corresponding eigenvector can be written with positive coordinates (see e.g. [32], pp. 53, 80). In the proof of Theorem 3 we saw that $|\lambda|>1$.

Since an irreducible matrix cannot have two linearly independent nonnegative eigenvectors (see [32], p. 63), all of the other eigenvectors must have a negative component. Thus, the stable eigenspace of the linearization does not intersect the nonnegative cone $R_{+}^{3}$. By the Stable Manifold Theorem for maps [33], the same holds true for the stable manifolds of $T_{1}$ and $T_{2}$ in some open neighborhood of $\hat{0}$. The lemma follows from the Hartman-Grobman Theorem for maps [33].

The following two lemmas follow directly from Lemma 3 and the fact that the only point mapped to $\hat{0}$ is $\hat{0}$.

Lemma 4 Let $b>b_{c r}$. For both $T_{1}$ and $T_{2}$, the set $M=\{\hat{0}\}$ is an isolated compact invariant set, i.e., $M$ is a compact forward invariant set and there exists a closed neighborhood $U \supset M$ in $R_{+}^{3}$ such that $M$ is the largest forward invariant set contained in $U$.

Lemma 5 Let $b>b_{c r}$. For both $T_{1}$ and $T_{2}$, the stable set of $M=\{\hat{0}\}$ is contained in $M$, i.e.,

$$
W^{s}(M) \doteq\left\{\hat{x}(0) \in R_{+}^{3} \mid \lim _{t \rightarrow \infty} \hat{x}(t)=\hat{0}\right\} \subseteq M .
$$

Lemmas 2, 4, and 5 imply the uniform persistence of $T_{1}$ and $T_{2}$ with respect to $M$ by the results in [39]:

Lemma 6 Let $b>b_{c r}$. Then $T_{1}$ and $T_{2}$ are uniformly persistent with respect to $M$, i.e., for each of $T_{1}$ and $T_{2}$ there exists an $\varepsilon>0$ such that $\liminf _{t \rightarrow \infty}\|\hat{x}(t)\|>\varepsilon$ for all orbits with $\hat{x}(0) \in R_{+}^{3}-M$.

Theorem 4 If $b>b_{c r}$, then the periodic LPA model (4) is uniformly persistent with respect to the extinction state $M$.

Proof. Let $b>b_{c r}$. Choose $\varepsilon>0$ as the smallest of the two $\varepsilon$ 's in Lemma 6 and let $\hat{x}(0) \in R_{+}^{3}-M$. Suppose $\liminf _{t \rightarrow \infty}\|\hat{x}(t)\| \leqq \varepsilon$, where $\hat{x}(t)$ is the orbit of $\hat{x}(0)$ under $T$. Then there exists a subsequence of points $\left\{\hat{x}\left(t_{j}\right)\right\}_{j=1}^{\infty}$ on the orbit with $\lim _{j \rightarrow \infty}\left\|\hat{x}\left(t_{j}\right)\right\| \leqq \varepsilon$. Now, infinitely many of the indices $t_{j}$ are even, or infinitely many are odd. Thus, there is a subsequence $\left\{\hat{x}_{i}\right\}_{i=1}^{\infty}$ of the orbit of $\hat{x}(0)$ under 
$T_{1}$ with $\lim _{i \rightarrow \infty}\left\|\hat{x}_{i}\right\| \leqq \varepsilon$, or there is a subsequence $\left\{\hat{y}_{i}\right\}_{i=1}^{\infty}$ of the orbit of $\hat{y}(0)=T(0, \hat{x}(0))$ under $T_{2}$ with $\lim _{i \rightarrow \infty}\left\|\hat{y}_{i}\right\| \leqq \varepsilon$. This violates Lemma 6 .

\section{Existence of 2-cycle solutions}

In this section we show the existence, uniqueness, and stability of positive 2-cycle solutions of the periodic LPA model (4), first for $b$ near $b_{c r}$ (with fixed $\alpha$ ), and then for small amplitude $\alpha$ (with fixed $b$ ).

\subsection{2-cycle solutions near $b=b_{c r}$}

Liapunov-Schmidt singular perturbation methods can be used to verify the existence and uniqueness of 2-periodic solutions of the periodic LPA model (4) for values of $b$ near $b_{c r}$. Henson [37] has shown existence, uniqueness, and stability properties of bifurcating periodic solutions of periodically-forced discrete dynamical systems. We will appeal to these results in the proof of the follow theorem.

Theorem 5 Let $0<\alpha<1$. For each $b>b_{\text {cr }}$ sufficiently close to $b_{c r}$, there exists a unique nontrivial 2-periodic solution $\hat{x}_{b}(t)=(L(t), P(t), A(t))$ of model (4) such that $\lim _{b \rightarrow b_{c r}} \hat{x}_{b}(t)=\hat{0}$.

Proof. This theorem follows from Theorem 2 in [37] once we have verified the hypotheses A1-A4 of that theorem.

A1: The equations in (4) can be expressed, in a straightforward way, as a single vector equation of the form

$$
\hat{x}(t+1)=b F(t, \hat{x}(t))+G(t, \hat{x}(t))
$$

where $F, G: N \times R^{3} \rightarrow R^{3}$, and both $F$ and $G$ are 2-periodic in $t$ and vanish at $\hat{x}=\hat{0}$. Here $\hat{x}=(L, P, A)$.

A2: For all $t \in N, F$ and $G$ are continuous functions of $\hat{x}$ on $R^{3}$ and are continuously Fréchet differentiable on $R^{3}$ with respect to $\hat{x}$.

A3-A4: Define $\Phi_{b, \hat{x}}$ to be the product of the Jacobians

$$
\Phi_{b, \hat{x}} \doteq \prod_{m=1}^{2}\left[b F^{\prime}(2-m, \hat{x}(2-m))+G^{\prime}(2-m, \hat{x}(2-m))\right] .
$$

Then

$$
\Phi_{b, 0}=\left(\begin{array}{ccc}
0 & b & b\left(1-\mu_{a}\right) \\
0 & 0 & b\left(1-\mu_{l}\right) \\
1-\mu_{l} & 1-\mu_{a} & \left(1-\mu_{a}\right)^{2}
\end{array}\right)
$$

It is easy to verify that $\Phi_{b_{c r}, 0}$ has a real eigenvalue of 1 and a nontrivial complex pair of eigenvalues lying inside the unit circle. Thus, $\operatorname{dim}\left[\operatorname{ker}\left(I-\Phi_{b_{c r}, 0}\right)\right]=1$. 
Since model (4) cannot have a nontrivial equilibrium solution, Theorem 5 refers to a 2-cycle of minimal period two. From the perspective of bifurcation theory, Theorem 5 says there is a transcritical bifurcation of nontrivial 2-cycle solutions at $b=b_{c r}$.

According to Theorem 3, the trivial solution 0 of model (4) is globally asymptotically stable if $b<b_{c r}$ and unstable if $b>b_{c r}$. The next theorem demonstrates a typical exchange of stability between the trivial solution and the bifurcating branch of 2-cycles at $b=b_{c r}$.

Theorem 6 If $b>b_{c r}$, then the nontrivial 2-periodic solution guaranteed by Theorem 5 is positive and (locally asymptotically) stable for $b$ sufficiently close to $b_{c r}$.

Proof. To prove this result we invoke Theorem 4 of [37], which first requires the verification of three hypotheses A5-A7.

A5: The second composite map of model (4) is a diffeomorphism in a neighborhood of $b_{c r}$ since $\Phi_{b_{c r}, 0}$ has nonzero eigenvalues.

A6: We must show:

$$
\frac{d}{d b}\left[\hat{l} \Phi_{b, 0} \hat{v}\right]_{b=b_{c r}} \neq 0
$$

where $\hat{l}$ and $\hat{v}$ are left and right eigenvectors of $\Phi_{b_{c}, 0}$ belonging to eigenvalue one such that $\hat{l} \hat{v}=1$. Straightforward calculations yield

$\hat{v}=\operatorname{col}\left(b_{c r}, \mu_{a}, 1\right) \doteq\left(\begin{array}{c}b_{c r} \\ \mu_{a} \\ 1\end{array}\right), \quad \hat{\imath}=\left(\begin{array}{llll}\frac{1-\mu_{l}}{1+2 \mu_{a}} & \frac{1}{1+2 \mu_{a}} & \frac{1}{1+2 \mu_{a}}\end{array}\right)$

and so

$$
\frac{d}{d b}\left[\hat{l} \Phi_{b, 0} \hat{v}\right]_{b=b_{c r}}=\frac{d}{d b}\left[\frac{2 b\left(1-\mu_{l}\right)+1}{1+2 \mu_{a}}\right]_{b=b_{c r}}=\frac{2\left(1-\mu_{l}\right)}{1+2 \mu_{a}} \neq 0 .
$$

A7: For all $t \in N, F$ and $G$ in (9) are twice continuously Fréchet differentiable in $\hat{x}$.

In order to determine the stability properties of the bifurcating 2-cycle solutions we consider the Liapunov-Schmidt expansions of $b$, the solution $\hat{x}$, and the dominant eigenvalue $\eta$ of $\Phi_{b, \hat{x}}$ as given by

$$
\begin{aligned}
& b=b_{c r}+\varepsilon b_{1}+O\left(\varepsilon^{2}\right) \\
& \hat{x}=\varepsilon \hat{v}+O\left(\varepsilon^{2}\right) \\
& \eta=1+\varepsilon \eta_{1}+O\left(\varepsilon^{2}\right) .
\end{aligned}
$$


The 2-cycle solutions are positive and stable for $b>b_{c r}$ sufficiently close to $b_{c r}$ if $b_{1}>0$ and $\eta_{1}<0$. From Theorem 4 of [37] we can calculate

$$
\begin{aligned}
& \eta_{1}=\frac{1}{2} \hat{l}\left(\begin{array}{ccc}
0 & 0 & b_{c r} \\
\left(1-\mu_{l}\right) & 0 & 0 \\
0 & 1 & \left(1-\mu_{a}\right)
\end{array}\right)\left(\begin{array}{ccc}
\frac{-c_{e l} b_{c r}}{1+\alpha} & 0 & \frac{-b_{c r}\left(b_{c r} c_{e l}+2 c_{e a}\right)}{1+\alpha} \\
0 & 0 & 0 \\
0 & \frac{-c_{p a}}{1+\alpha} & \frac{-c_{p a} \mu_{a}}{1+\alpha}
\end{array}\right) \hat{v} \\
& +\frac{1}{2} \hat{l}\left(\begin{array}{ccc}
\frac{-c_{e l} b_{c r}}{1-\alpha} & 0 & \frac{-b_{c r}\left(b_{c r} c_{e l}+2 c_{e a}\right)}{1-\alpha} \\
0 & 0 & 0 \\
0 & \frac{-c_{p a}}{1-\alpha} & \frac{-c_{p a} \mu_{a}}{1-\alpha}
\end{array}\right) \hat{v} \\
& =\frac{-2 \mu_{a}}{\left(1-\alpha^{2}\right)\left(1+2 \mu_{a}\right)}\left(c_{e a}+b_{c r} c_{e l}+c_{p a}\right)<0
\end{aligned}
$$

and

$$
b_{1}=-\frac{\eta_{1}}{\frac{d}{d b}\left[\hat{l} \Phi_{b, 0} \hat{v}\right]_{b=b_{c r}}}=\frac{b_{c r}}{\left(1-\alpha^{2}\right)}\left(c_{e a}+b_{c r} c_{e l}+c_{p a}\right)>0 .
$$

\subsection{Small amplitude 2-cycles}

We now show the existence of small amplitude solutions for "generic" values of $b$. Let $J$ be the Jacobian of $T$.

Theorem 7 Let $b>b_{c r}$ and $\hat{x}_{0}=\left(L_{0}, P_{0}, A_{0}\right)$ be the unique positive equilibrium solution of the LPA model (1). Assume the matrix $I-J^{2}\left(\hat{x}_{0}\right)$ is nonsingular. Then for each sufficiently small $\alpha>0$ there exists a unique 2-cycle solution $\hat{x}_{\alpha}(t)$ of the periodic LPA model (4) which is positive and for which $\lim _{\alpha \rightarrow 0} \hat{x}_{\alpha}(t)=\hat{x}_{0}$. For sufficiently small $\alpha>0$, this 2-cycle is (locally asymptotically) stable or unstable if the corresponding equilibrium $\hat{x}_{0}$ is (locally asymptotically) stable or unstable respectively. The components of $\hat{x}_{\alpha}(t)$ are infinitely differentiable in $\alpha$ for each $t \geqq 0$.

Proof. Denote the periodic LPA model (4) by

$$
\hat{x}(t+1)=K(t, \alpha, \hat{x}(t))
$$

where $\hat{x}=(L, P, A)$. We look for 2-cycle solutions of the form

$$
\hat{x}_{\alpha}(t)=\hat{x}_{0}+\hat{y}(t, \alpha)
$$

where $\hat{x}_{0}=\left(L_{0}, P_{0}, A_{0}\right)$, and $\hat{y}$ is 2-periodic in $t$ and vanishes at $\alpha=0$. Model (4) can then be equivalently expressed as

$$
\hat{y}(t+1, \alpha)=J\left(\hat{x}_{0}\right) \hat{y}(t, \alpha)+H(t, \alpha, \hat{y}(t, \alpha))+K\left(t, \alpha, \hat{x}_{0}\right)-\hat{x}_{0}
$$


where $J$ is the Jacobian, $H$ is $O\left(\|\hat{y}\|^{2}\right)$, and $K\left(t, 0, \hat{x}_{0}\right)=\hat{x}_{0}$ for all $t \geqq 0$. Note that for $\alpha>0$, equation (13) does not admit the trivial solution $\hat{y} \equiv \hat{0}$ since $\hat{x}_{0}$ cannot be an equilibrium solution of the periodic model (4).

There exists a 2-cycle solution $\hat{y}(t, \alpha)$ of equation (13) if and only if there exists a solution $\hat{z} \in R^{3}$ of

$$
\Omega(\hat{z}, \alpha) \doteq \hat{z}-\Psi(\hat{z}, \alpha)=\hat{0}
$$

where

$$
\begin{aligned}
\Psi(\hat{z}, \alpha) \doteq & \left(I-J^{2}\left(\hat{x}_{0}\right)\right)^{-1}\left\{J\left(\hat{x}_{0}\right)\left[H(0, \alpha, \hat{z})+K\left(0, \alpha, \hat{x}_{0}\right)-\hat{x}_{0}\right]\right. \\
& \left.+H\left(1, \alpha,\left[J\left(\hat{x}_{0}\right) \hat{z}+H(0, \alpha, \hat{z})+K\left(0, \alpha, \hat{x}_{0}\right)-\hat{x}_{0}\right]\right)+K\left(1, \alpha, \hat{x}_{0}\right)-\hat{x}_{0}\right\} .
\end{aligned}
$$

Now,

$$
\Omega(\hat{0}, 0)=\hat{0} \quad \text { and }\left.\quad \frac{\partial \Omega}{\partial \hat{z}}\right|_{z=\alpha=0}=I
$$

since $H$ is $O\left(\|\hat{z}\|^{2}\right)$. By the Implicit Function Theorem, for sufficiently small $\alpha$ there exists a unique $\hat{z}(\alpha)$ such that $\Omega(\hat{z}(\alpha), \alpha)=\hat{0}, \hat{z}(0)=\hat{0}$, and the components of $\hat{z}$ are infinitely differentiable in $\alpha$. Therefore, for each sufficiently small $\alpha$ there exists a unique nontrivial 2-cycle solution $\hat{x}_{\alpha}(t)=\hat{x}_{0}+\hat{y}(t, \alpha)$ of model (4) which is defined by $\hat{y}(0, \alpha) \doteq \hat{z}(\alpha)$ and whose components are infinitely differentiable in $\alpha$ for each $t \geqq 0$.

If $\hat{x}_{0}$ is a stable (unstable) equilibrium of the LPA model (1), then it is also a stable (unstable) 2-cycle solution of the periodic LPA model (4) for $\alpha=0$; thus the 2-cycle solution $\hat{x}(t)=\hat{x}_{0}+\hat{y}(t, \alpha)$ of model (4) is stable (unstable) for small $\alpha$ by a continuity argument.

In Theorem 7, the generic hypotheses that $I-J^{2}\left(\hat{x}_{0}\right)$ be nonsingular is satisfied at all hyperbolic equilibria $\hat{x}_{0}$.

\section{The effect of environmental periodicity}

Let $(L(t), P(t), A(t))$ be a 2-cycle solution of the periodic LPA model (4). Define the time average

$$
\tau \doteq\langle L+P+A\rangle \doteq \frac{1}{2} \sum_{t=o}^{1}(L(t)+P(t)+A(t))
$$

and consider the "average solution surface" $\Sigma$ defined by $\tau=\tau(\alpha, b)$. Figure 1 shows this surface for the parameter estimates obtained from Jillson's data [40]. The surface $\Sigma$ bifurcates from the $\alpha-b$ plane along the line $b=b_{c r}$, and the equilibria of the LPA model (1) lie in the $\alpha=0$ coordinate plane. Graphically, we see from Fig. 1 that a positive (negative) effect of environmental periodicity occurs at points $(\alpha, b)$ in the plane above which the surface $\Sigma$ is higher (lower) than at the corresponding equilibrium value on the surface above the point $(0, b)$. For example, in Fig. 1 a positive effect is clearly present at $\alpha=0.5$, $b=1.0$ and a negative effect is present at $\alpha=0.5, b=0.4$. 
Simulations (such as Figs. 1 and 2) suggest the periodic LPA model (4) can exhibit both positive and negative effects; however, analytic verification is nontrivial. We now turn our attention to this problem.

\subsection{Near the primary bifurcation line $b=b_{c r}$}

We first show that a negative effect of environmental periodicity always occurs near the primary bifurcation point $b=b_{c r}$ for any amplitude $\alpha$.

Theorem 8 Let $b>b_{c r}, \alpha \in(0,1)$, and let $\left(L_{0}, P_{0}, A_{0}\right)$ be the unique positive equilibrium solution of the LPA model (1). Let $\left(L_{\alpha}(t), P_{\alpha}(t), A_{\alpha}(t)\right)$ be the nontrivial 2-cycle solution of the periodic LPA model (4) guaranteed by Theorem 5.

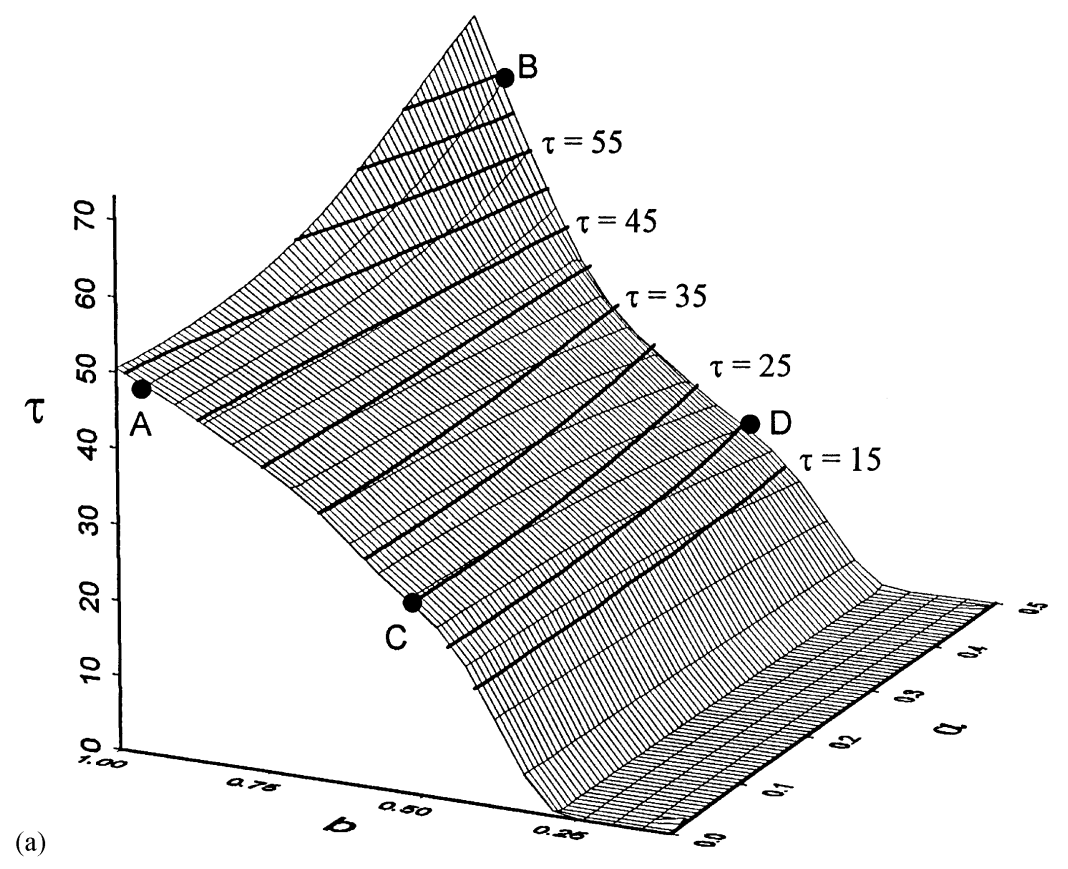

Fig. 1a. The surface $\Sigma$ defined by the average total population size $\tau=\tau(b, \alpha)$ as a function of the amplitude $\alpha$ of environmental periodicity and the inherent larval recruitment rate $b$ is shown for the periodic LPA model (4). This surface was drawn using parameter set 1 in Table 1 (obtained from Jillson's data [40]). On the surface are drawn the $\alpha-b$ coordinate lines (finer lines) which can be seen crossing the $\tau$ darker level surface contour lines (shown for $\tau=15$ to $60 \mathrm{in}$ increments of 5). The path from point $A$ to point $B$ with fixed $b$ crosses level surface curves corresponding to increasing values of $\tau$ (from under 50 to nearly 60). This illustrates a positive effect of environmental periodicity as the amplitude increases from $\alpha=0.0$ to 0.5 . The path from point $C$ to point $D$ with fixed $b$ crosses level surface curves corresponding to decreasing values of $\tau$ (from over 25 to under 20). This illustrates a negative effect of environmental periodicity as the amplitude increases from $\alpha=0.0$ to 0.5 


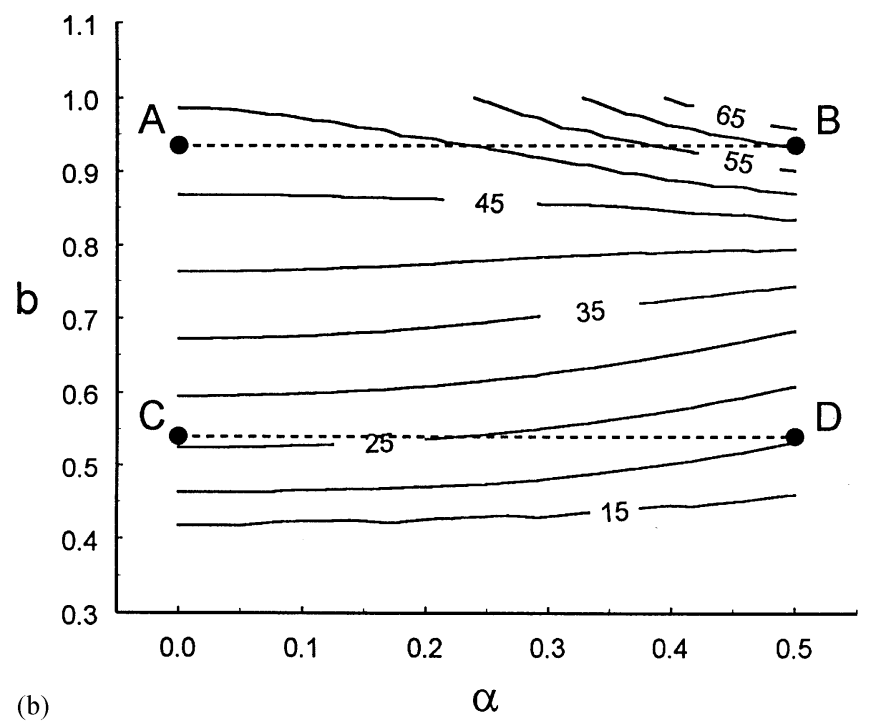

Fig. 1b. The contour map of the surface $\Sigma$ in (a) is shown in the $\alpha-b$ plane. The (dotted) straight line paths from points $A$ to $B$ and from points $C$ to $D$ are the projections of the corresponding paths shown on the surface $\Sigma$ in Fig. 1a. The path from $A$ to $B$ crosses contours of increasing values of the average total population $\tau$ while that from $C$ to $D$ crosses contours of decreasing values

Then for $b$ sufficiently close to $b_{c r}$ and all $\alpha \in(0,1)$ we have the inequalities

$$
\begin{gathered}
\left\langle L_{\alpha}(t)\right\rangle<L_{0} \\
\left\langle P_{\alpha}(t)\right\rangle<P_{0} \\
\left\langle A_{\alpha}(t)\right\rangle<A_{0} .
\end{gathered}
$$

Proof. By equation (11), the Liapunov-Schmidt expansion for the 2-cycle solution of the periodic LPA model is, for small $\varepsilon_{p}>0$,

$$
\begin{aligned}
& L_{\alpha}(t)=\varepsilon_{p} b_{c r}+O\left(\varepsilon_{p}^{2}\right)(t) \\
& P_{\alpha}(t)=\varepsilon_{p} \mu_{c r}+O\left(\varepsilon_{p}^{2}\right)(t) \\
& A_{\alpha}(t)=\varepsilon_{p}+O\left(\varepsilon_{p}^{2}\right)(t)
\end{aligned}
$$

and

$$
b=b_{c r}+\varepsilon_{p}\left(\frac{b_{c r}\left(c_{e a}+b_{c r} c_{e l}+c_{p a}\right)}{\left(1-\alpha^{2}\right)}\right)+O\left(\varepsilon_{p}^{2}\right) .
$$

The Liapunov-Schmidt methods of [37] can also be used to find expansions for the equilibrium solutions of the autonomous LPA model as a periodically-forced model with forcing of period one. Straightforward 

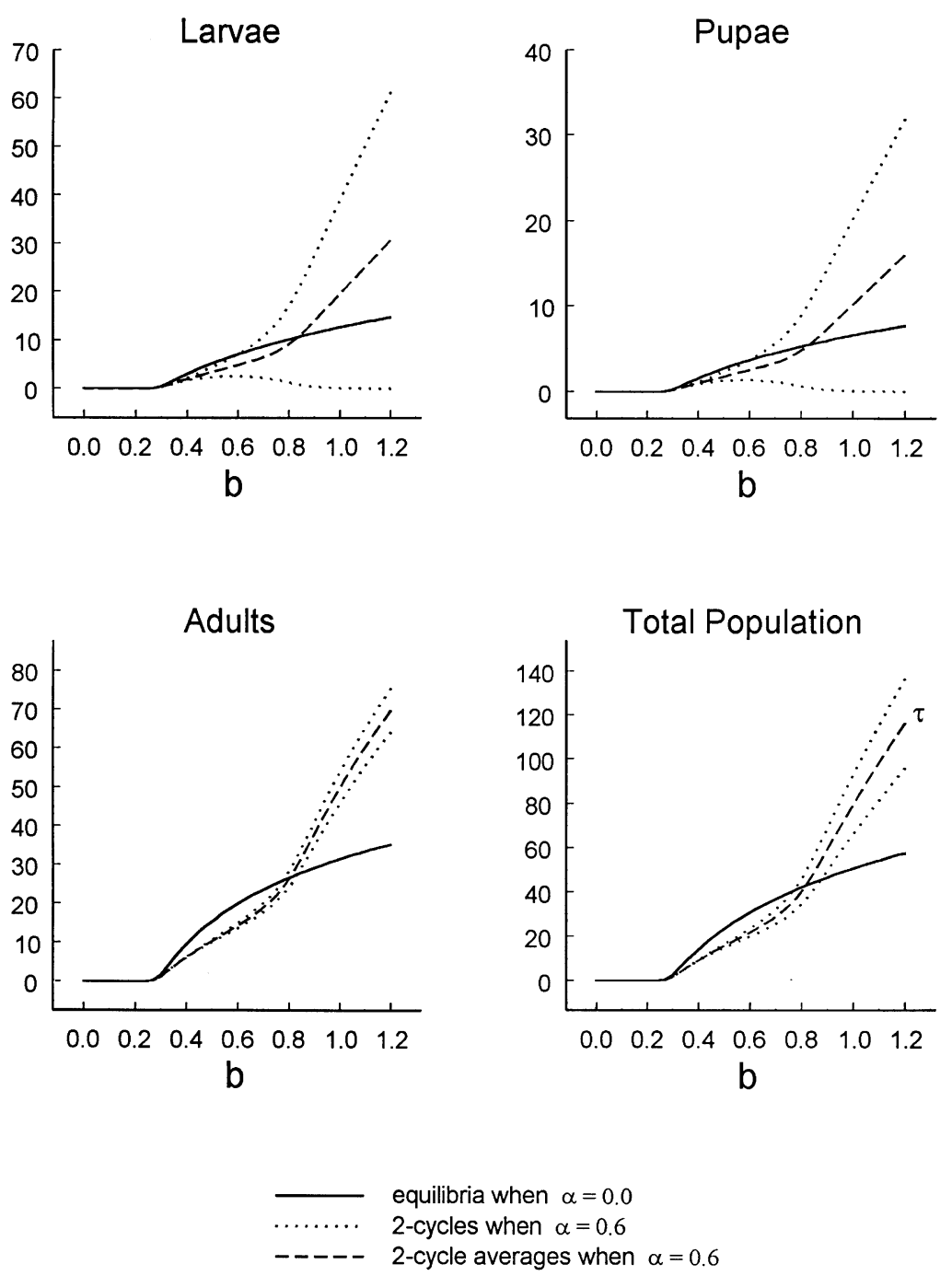

Fig. 2. The asymptotic states of both the autonomous LPA model (1) and the periodic LPA model (4) are shown plotted against the parameter $b$ for the parameter set 1 in Table 1 (obtained from Jillson's data [40]). All three life cycle stages $L, P$ and $A$ are shown as well as the total population size $L+P+A$. In the periodic case the relative amplitude $\alpha=0.6$ is used (the same as used by Jillson [40] ). The solid line shows, for the autonomous model, the bifurcation of positive equilibria from the trivial (extinction) equilibrium at a critical value of $b_{c r}=\mu_{a} /\left(1-\mu_{l}\right) \approx 0.293$. The dotted line shows, for the periodic model, the bifurcation of positive 2-cycles at the same critical value of $b$. The dashed line is the (time) average of the bifurcating positive 2-cycles. (The total population cycle average $\tau$ is the same as that used to construct the surface $\Sigma$ in Fig. 1a.) These averages (indeed, even the maxima of the 2-cycles) are clearly seen to be less than the equilibrium level of the autonomous model for smaller values of $b>b_{c}$, as predicted by Theorem 8 . For larger values of $b$, however, the cycle averages cross over and exceed the equilibrium level of the autonomous model, as predicted by Theorem 9 
calculations show, for small $\varepsilon_{a}>0$, that

$$
\begin{aligned}
L_{0} & =\varepsilon_{a} b_{c r}+O\left(\varepsilon_{a}^{2}\right) \\
P_{0} & =\varepsilon_{a} \mu_{a}+O\left(\varepsilon_{a}^{2}\right) \\
A_{0} & =\varepsilon_{a}+O\left(\varepsilon_{a}^{2}\right) \\
b & =b_{c r}+\varepsilon_{a} b_{c r}\left(c_{e a}+b_{c r} c_{e l}+c_{p a}\right)+O\left(\varepsilon_{a}^{2}\right) .
\end{aligned}
$$

In order to compare the solutions to first order at the same values of $b$, we require

Then

$$
\varepsilon_{p}=\varepsilon_{a}\left(1-\alpha^{2}\right)
$$

$$
\begin{aligned}
& L_{0}-\left\langle L_{\alpha}(t)\right\rangle=\varepsilon_{a} \alpha^{2} b_{c r}+O\left(\varepsilon_{a}^{2}\right)>0 \\
& P_{0}-\left\langle P_{\alpha}(t)\right\rangle=\varepsilon_{a} \alpha^{2} \mu_{a}+O\left(\varepsilon_{a}^{2}\right)>0 \\
& A_{0}-\left\langle A_{\alpha}(t)\right\rangle=\varepsilon_{a} \alpha^{2}+O\left(\varepsilon_{a}^{2}\right)>0
\end{aligned}
$$

for $\varepsilon_{a}>0$ sufficiently small.

Theorem 8 implies that for small larval recruitment rates $b>b_{c r}$ the effect of environmental periodicity is negative on all three life cycle stages. It follows then that the effect of environmental periodicity is negative for total population numbers. This result implies that if positive effects are to occur it must be for "large" larval recruitment values $b$. In the next section we turn our attention to large values of $b$.

\subsection{Near the equilibrium branch}

In this section we consider the periodic LPA model for large values of the larval recruitment rate $b$. We do this, however, only for small values of the relative amplitude $\alpha$.

Let $I \subseteq\left(b_{c r}, \infty\right)$ be a "hyperbolic interval", i.e. an interval such that the equilibrium $\left(L_{0}, P_{0}, A_{0}\right)$ is hyperbolic whenever $b \in I$. Then by Theorem 7 we can write the small amplitude 2-cycle solutions of the periodic LPA model as:

$$
\begin{aligned}
& L(t+1)=L_{0}+L_{1}(t) \alpha+L_{2}(t) \alpha^{2}+O\left(\alpha^{3}\right)(t) \\
& P(t+1)=P_{0}+P_{1}(t) \alpha+P_{2}(t) \alpha^{2}+O\left(\alpha^{3}\right)(t) \\
& A(t+1)=A_{0}+A_{1}(t) \alpha+A_{2}(t) \alpha^{2}+O\left(\alpha^{3}\right)(t)
\end{aligned}
$$

where the $\alpha$-coefficients are 2-periodic in $t$.

Equations for the first and second order terms $L_{1}(t), L_{2}(t)$, etc. can be found by a substitution of these expansions into the periodic LPA model equations (4) and equating coefficients of like powers of $\alpha$ from both sides of the resulting equations. From the first order terms we obtain equations for 
$L_{1}(t)$ and $A_{1}(t)$ that can be written in the form

$$
\begin{aligned}
& \frac{L_{1}(t+1)}{L_{0}}=\frac{A_{1}(t)}{A_{0}}+c_{e a}\left[(-1)^{t} A_{0}-A_{1}(t)\right]+c_{e l}\left[(-1)^{t} L_{0}-L_{1}(t)\right] \\
& \frac{A_{1}(t+1)}{A_{0}}=\mu_{a} \frac{L_{1}(t+1)}{L_{0}}+\mu_{a} c_{p a}\left[(-1)^{t} A_{0}-A_{1}(t)\right]+\left(1-\mu_{a}\right) \frac{A_{1}(t)}{A_{0}} .
\end{aligned}
$$

By averaging both sides of these equations we obtain the linear, homogeneous system of algebraic equations

$$
\begin{array}{r}
\left(c_{e a}-\frac{1}{A_{0}}\right)\left\langle A_{1}\right\rangle+\left(c_{e l}+\frac{1}{L_{0}}\right)\left\langle L_{1}\right\rangle=0 \\
\left(\mu_{a} c_{p a}+\frac{\mu_{a}}{A_{0}}\right)\left\langle A_{1}\right\rangle-\left(\frac{\mu_{a}}{L_{0}}\right)\left\langle L_{1}\right\rangle=0
\end{array}
$$

for the averages $\left\langle A_{1}\right\rangle$ and $\left\langle L_{1}\right\rangle$. Hence $\left\langle A_{1}\right\rangle=\left\langle L_{1}\right\rangle=0$ as long as the coefficient matrix

$$
\left(\begin{array}{cc}
c_{e a}-\frac{1}{A_{0}} & c_{e l}+\frac{1}{L_{0}} \\
\mu_{a} c_{p a}+\frac{\mu_{a}}{A_{0}} & \frac{\mu_{a}}{L_{0}}
\end{array}\right)
$$

is nonsingular, which is true for large $b$ since its determinant approaches the nonzero value $-\mu_{a} c_{p a} c_{e l}$ as $b \rightarrow \infty$. It follows that in order to determine the relationship of the 2-cycle averages

$$
\begin{aligned}
& \langle L\rangle=L_{0}+\left\langle L_{2}\right\rangle \alpha^{2}+O\left(\alpha^{3}\right) \\
& \langle P\rangle=P_{0}+\left\langle P_{2}\right\rangle \alpha^{2}+O\left(\alpha^{3}\right) \\
& \langle A\rangle=A_{0}+\left\langle A_{2}\right\rangle \alpha^{2}+O\left(\alpha^{3}\right)
\end{aligned}
$$

to the corresponding equilibrium values $L_{0}, P_{0}, A_{0}$ for large values of $b$, it is necessary to calculate the averages $\left\langle L_{2}\right\rangle,\left\langle P_{2}\right\rangle,\left\langle A_{2}\right\rangle$ of the second order coefficients $L_{2}(t), P_{2}(t)$, and $A_{2}(t)$. To do this we will need some further facts about the first order coefficients for large values of $b$.

Since the averages $\left\langle A_{1}\right\rangle$ and $\left\langle L_{1}\right\rangle$ both equal 0 , it follows that

$$
\begin{aligned}
& A_{1}(1)=-A_{1}(0) \\
& L_{1}(1)=-L_{1}(0) .
\end{aligned}
$$

From these facts and from equations (15) and (16) we can calculate the formula

$$
A_{1}(0)=\frac{\mu_{a} A_{0}\left(c_{e a} A_{0}+c_{e l} L_{0}\right)-c_{p a} \mu_{a} A_{0}^{2}\left(c_{e l} L_{0}-1\right)}{\left(2-c_{p a} \mu_{a} A_{0}-\mu_{a}\right)\left(c_{e l} L_{0}-1\right)+\mu_{a}\left(c_{e a} A_{0}-1\right)} .
$$


Recall that the equilibria are unbounded, strictly increasing functions of $b$ (Theorem 1), and that

$$
\lim _{b \rightarrow \infty} \frac{A_{0}}{L_{0}}=0
$$

by equation (3). From equation (17) we see that

and hence

$$
\lim _{b \rightarrow \infty} \frac{A_{1}(0)}{A_{0}}=1
$$

$$
\lim _{b \rightarrow \infty} \frac{A_{1}(t)}{A_{0}}=(-1)^{t}
$$

From equation (15) we obtain (after setting $t=0$, dividing both sides by $L_{0}$, and rearranging terms)

$$
\frac{L_{1}(0)}{L_{0}}=\left(\frac{L_{0}}{c_{e l} L_{0}-1}\right)\left[\frac{A_{1}(0)}{A_{0} L_{0}}+c_{e a}\left(\frac{A_{0}}{L_{0}}-\frac{A_{1}(0)}{A_{0}} \frac{A_{0}}{L_{0}}\right)+c_{e l}\right]
$$

and so

$$
\lim _{b \rightarrow \infty} \frac{L_{1}(t)}{L_{0}}=(-1)^{t} .
$$

Finally, from equation (16) we can therefore calculate the limit

$$
\lim _{b \rightarrow \infty}\left[(-1)^{t} A_{0}-A_{1}(t)\right]=\frac{-2\left(1-\mu_{a}\right)(-1)^{t}}{\mu_{a} c_{p a}}
$$

and, using this in equation (15), the limit

$$
\lim _{b \rightarrow \infty}\left[(-1)^{t} L_{0}-L_{1}(t)\right]=\left[\frac{2 c_{e a}\left(1-\mu_{a}\right)(-1)^{t}}{\mu_{a} c_{p a} c_{e l}}\right]-\frac{2(-1)^{t}}{c_{e l}} .
$$

We are now ready to calculate the second order averages $\left\langle L_{2}\right\rangle,\left\langle P_{2}\right\rangle$, and $\left\langle A_{2}\right\rangle$ for large values of $b$. From the equations for the second order coefficients obtained by the substitution of the expansions (14) into the periodic LPA model we obtain the two equations

$$
\begin{aligned}
& \frac{L_{2}(t+1)}{L_{0}}-\frac{A_{2}(t)}{A_{0}}+c_{e a} A_{2}(t)+c_{e l} L_{2}(t) \\
& =\left[\frac{A_{1}(t)}{A_{0}}-(-1)^{t}\right]\left\{c_{e a}\left[(-1)^{t} A_{0}-A_{1}(t)\right]+c_{e l}\left[(-1)^{t} L_{0}-L_{1}(t)\right]\right\} \\
& \quad+\frac{1}{2}\left\{c_{e a}\left[(-1)^{t} A_{0}-A_{1}(t)\right]+c_{e l}\left[(-1)^{t} L_{0}-L_{1}(t)\right]\right\}^{2}
\end{aligned}
$$




$$
\begin{aligned}
& \frac{A_{2}(t+1)}{A_{0}}-\mu_{a} \frac{L_{2}(t+1)}{L_{0}}+\mu_{a} c_{p a} A_{2}(t)-\left(1-\mu_{a}\right) \frac{A_{2}(t)}{A_{0}} \\
& =\mu_{a} c_{p a}\left[\frac{L_{1}(t+1)}{L_{0}}-(-1)^{t}\right]\left[(-1)^{t} A_{0}-A_{1}(t)\right] \\
& +\mu_{a} c_{p a}^{2}\left[(-1)^{t} A_{0}-A_{1}(t)\right]^{2}
\end{aligned}
$$

for $L_{2}(t)$ and $A_{2}(t)$. From the first equation (18) we obtain the limit

$$
\lim _{b \rightarrow \infty}\left[\frac{L_{2}(t+1)}{L_{0}}-\frac{A_{2}(t)}{A_{0}}+c_{e a} A_{2}(t)+c_{e l} L_{2}(t)\right]=\frac{1}{2}\left[-2(-1)^{t}\right]^{2}=2 .
$$

From the second equation (19) we obtain the limit

$$
\begin{aligned}
\lim _{b \rightarrow \infty} & {\left[\frac{A_{2}(t+1)}{A_{0}}-\mu_{a} \frac{L_{2}(t+1)}{L_{0}}+\mu_{a} c_{p a} A_{2}(t)-\left(1-\mu_{a}\right) \frac{A_{2}(t)}{A_{0}}\right] } \\
& =-2 \mu_{a} c_{p a}(-1)^{t}\left[\frac{-2\left(1-\mu_{a}\right)(-1)^{t}}{\mu_{a} c_{p a}}\right]+\mu_{a} c_{p a}^{2}\left[\frac{-2\left(1-\mu_{a}\right)(-1)^{t}}{\mu_{a} c_{p a}}\right]^{2} \\
& =4\left(1-\mu_{a}\right)+\frac{4\left(1-\mu_{a}\right)^{2}}{\mu_{a}} \\
& =\frac{4\left(1-\mu_{a}\right)}{\mu_{a}} .
\end{aligned}
$$

An averaging of equations (20) and (21) yields the limits

$$
\begin{aligned}
& \lim _{b \rightarrow \infty} {\left[\left(c_{e a}-\frac{1}{A_{0}}\right)\left\langle A_{2}\right\rangle+\left(c_{e l}+\frac{1}{L_{0}}\right)\left\langle L_{2}\right\rangle\right]=2 } \\
& \lim _{b \rightarrow \infty}\left[\left(\mu_{a} c_{p a}+\frac{\mu_{a}}{A_{0}}\right)\left\langle A_{2}\right\rangle-\left(\frac{\mu_{a}}{L_{0}}\right)\left\langle L_{2}\right\rangle\right]=\frac{4\left(1-\mu_{a}\right)}{\mu_{a}}
\end{aligned}
$$

Thus, the pair $\left(\left\langle A_{2}\right\rangle,\left\langle L_{2}\right\rangle\right)$ can be made arbitrarily close to the solution $(x, y)$ of the linear system of equations

$$
\begin{aligned}
c_{e a} x+c_{e l} y & =2 \\
\mu_{a} c_{p a} x & =\frac{4\left(1-\mu_{a}\right)}{\mu_{a}}
\end{aligned}
$$

given sufficiently large $b$. That is,

$$
\begin{aligned}
& \lim _{b \rightarrow \infty}\left\langle A_{2}\right\rangle=\frac{4\left(1-\mu_{a}\right)}{\mu_{a}^{2} c_{p a}}>0 \\
& \lim _{b \rightarrow \infty}\left\langle L_{2}\right\rangle=\frac{2}{c_{e l}}-\frac{4 c_{e a}\left(1-\mu_{a}\right)}{\mu_{a}^{2} c_{p a} c_{e l}}
\end{aligned}
$$


Now, $\lim _{b \rightarrow \infty}\left\langle A_{2}\right\rangle$ is clearly positive, but the sign of $\lim _{b \rightarrow \infty}\left\langle L_{2}\right\rangle$ is positive if and only if

$$
\frac{4 c_{e a}\left(1-\mu_{a}\right)}{\mu_{a}^{2} c_{p a}}<2
$$

Finally, from these formulas for the 2-cycle averages of each life cycle stage we can obtain a formula for the average $\tau$ of the total population numbers $T(t)=L(t)+P(t)+A(t)$, namely

where

$$
\tau=L_{0}+P_{0}+A_{0}+\left\langle L_{2}+P_{2}+A_{2}\right\rangle \alpha^{2}+O\left(\alpha^{3}\right)
$$

$$
\begin{aligned}
\lim _{b \rightarrow \infty}\left\langle L_{2}+P_{2}+A_{2}\right\rangle= & {\left[\frac{2}{c_{e l}}-\frac{4 c_{e a}\left(1-\mu_{a}\right)}{\mu_{a}^{2} c_{p a} c_{e l}}\right] } \\
& +\left(1-\mu_{l}\right)\left[\frac{2}{c_{e l}}-\frac{4 c_{e a}\left(1-\mu_{a}\right)}{\mu_{a}^{2} c_{p a} c_{e l}}\right]+\frac{4\left(1-\mu_{a}\right)}{\mu_{a}^{2} c_{p a}} \\
= & \left(2-\mu_{l}\right)\left[\frac{2}{c_{e l}}-\frac{4 c_{e a}\left(1-\mu_{a}\right)}{\mu_{a}^{2} c_{p a} c_{e l}}\right]+\frac{4\left(1-\mu_{a}\right)}{\mu_{a}^{2} c_{p a}} .
\end{aligned}
$$

From the above calculations, after some algebraic manipulations, we deduce the following theorem.

Theorem 9 Let $b \in I \subseteq\left(b_{c r},+\infty\right)$ where $I$ is a hyperbolic interval, and let $(L(t), P(t), A(t))$ be the 2-cycle solution of the periodic LPA model guaranteed by Theorem 7 for sufficiently small $\alpha$. Then the following statements hold for sufficiently large larval recruitment rates $b$ and sufficiently small relative amplitudes $\alpha>0$.

$$
\begin{gathered}
\langle A(t)\rangle>A_{0} \\
\langle L(t)\rangle>L_{0} \quad \text { and }\langle P(t)\rangle>P_{0} \quad \text { if } \frac{\mu_{a}^{2}}{1-\mu_{a}}>2 \frac{c_{e a}}{c_{p a}} \\
\langle L(t)\rangle<L_{0} \quad \text { and }\langle P(t)\rangle<P_{0} \quad \text { if } \frac{\mu_{a}^{2}}{1-\mu_{a}}<2 \frac{c_{e a}}{c_{p a}} \\
\tau>\tau_{0} \quad \text { if } \frac{\mu_{a}^{2}}{1-\mu_{a}}>2 \frac{c_{e a}}{c_{p a}}-\left(\frac{2}{2-\mu_{l}}\right) \frac{c_{e l}}{c_{p a}} \\
\tau<\tau_{0} \quad \text { if } \frac{\mu_{a}^{2}}{1-\mu_{a}}<2 \frac{c_{e a}}{c_{p a}}-\left(\frac{2}{2-\mu_{l}}\right) \frac{c_{e l}}{c_{p a}}
\end{gathered}
$$

where $\tau=\langle L(t)+P(t)+A(t)\rangle$ and $\tau_{0}=L_{0}+P_{0}+A_{0}$.

The first conclusion to be drawn from this theorem is that positive effects on total population numbers due to environmental periodicities are possible in the periodic LPA model. For example, the inequality

$$
\frac{\mu_{a}^{2}}{1-\mu_{a}}>2 \frac{c_{e a}}{c_{p a}}-\left(\frac{2}{2-\mu_{l}}\right) \frac{c_{e l}}{c_{p a}}
$$


holds for adult death rates $\mu_{a}$ sufficiently close to 1 (or for all $\mu_{a} \in(0,1)$ if the right hand side is negative). Another conclusion is the possibility for different life cycle stages to be affected differently. The inequality

$$
\frac{\mu_{a}^{2}}{1-\mu_{a}}>2 \frac{c_{e a}}{c_{p a}}
$$

implies the inequality (22), but not conversely. Thus, it is possible for the larval and pupal stages to be negatively effected by environmental periodicity while the adult stage and the total population numbers are positively effected.

\section{Concluding remarks}

Motivated by experimental results reported by Jillson [40] in which cultures of flour beetles (Tribolium) showed striking increases in population numbers when their flour habitat was periodically oscillated in volume, we have introduced the periodically forced version (4) of a three stage model for flour beetle dynamics that takes into account periodically fluctuating volumes of flour. This model is a modification of a autonomous model (1) for flour beetle dynamics that has been statistically validated by means of controlled and replicated laboratory experiments $[14,25]$. The success of the autonomous model equations (1) in predicting flour beetle dynamics and their bifurcations in a constant habitat [28, 14] suggests an appropriate modification might predict the effects of a periodically fluctuating habitat on beetle dynamics, including the observations of Jillson. The periodic version of the model is derived under the assumption that habitat fluctuations effect only the cannibalism rates $[13,54]$. This assumption has been recently validated by laboratory experiments [16].

Several fundamental mathematical facts about the periodically forced system of difference equations (4) are given. First of all, the uniform persistence result in Theorem 4 shows that environmental periodicities do not lead to extinctions when the larval recruitment rate $b$ exceeds a critical value $b_{c r}$.

Second, Theorems 6 and 7 provide two situations in which it can be proved mathematically that period 2 forcing gives rise to 2 -cycle solutions (i.e. to period 2 oscillations in all life cycle stages) :

(1) all values of the relative amplitude $\alpha \in(0,1)$, but larval recruitment rates $b$ close to (but larger than) the critical value $b_{c r}$;

(2) all ("generic") values of $b>b_{c r}$, but small values of the relative amplitude $\alpha$.

Third, Theorems 8 and 9 address the effect of periodic forcing by comparing the averages of the 2-cycle solution of the periodically forced model (4) to the equilibrium levels of the autonomous model (1). These theorems show that environmental periodicity in the periodic LPA model can have either negative or positive effects, depending upon parameters values. In case (1), for small larval recruitment rates $b$, the effect is always negative; the averages of all three 
Table 1. Five sets of model parameter estimates are presented. Parameter set 1 was obtained from Jillson's experimental data (T. castenum, sensitive strain) [40]. Parameter sets 2 and 3 (T. castenum, RR strain) and sets 4 and 5 (T. castenum, SS strain) were obtained from [16] (also see [14] ). All five sets satisfy the inequality (22). Nontrivial positive equilibria of the autonomous LPA model equations (1) exist for $b>b_{c r}=\mu_{a} /\left(1-\mu_{l}\right)$. For relative amplitude $\alpha$ a periodic volume of flour has a negative effect for larval recruitment rates $b<b_{\alpha}$ and a positive effect for $b>b_{\alpha}$. A “*” indicates that the "cross over" point $b_{\alpha}$ is greater than the equilibrium destabilization point

\begin{tabular}{llllllllll}
\hline & $\mu_{a}$ & $\mu_{l}$ & $c_{e l}$ & $c_{e a}$ & $c_{p a}$ & $b$ & $b_{c r}$ & $b_{0.1}$ & $b_{0.6}$ \\
\hline 1 & 0.1524 & 0.4794 & 0.0584 & 0.0058 & 0.0105 & 4.445 & 0.293 & 0.876 & 0.828 \\
2 & 0.7300 & 0.1612 & 0.0138 & 0.0111 & 0.0043 & 7.876 & 0.870 & 5.285 & 5.365 \\
3 & 0.9600 & 0.1612 & 0.0138 & 0.0111 & 0.0043 & 7.876 & 1.145 & $*$ & $*$ \\
4 & 0.7300 & 0.2670 & 0.0109 & 0.0090 & 0.0040 & 7.880 & 0.996 & 6.055 & 6.075 \\
5 & 0.9600 & 0.2670 & 0.0109 & 0.0090 & 0.0040 & 7.880 & 1.310 & $*$ & $*$ \\
\hline
\end{tabular}

life cycle stages, and hence that of the total population numbers, are reduced in the periodic habitat. (See Fig. 2.) In case (2) however, for large larval recruitment rates $b>b_{c r}$, small amplitude environmental periodicities always have a positive effect on the adult stage in the periodic LPA model. Larval and pupal numbers and total population numbers (the sum of all larvae, pupae and adults) may or may not be positively effected, however, depending upon model parameter values as described in Theorem 9. For example, it can be seen from the inequalities (22) and (23) that large adult death rates $\mu_{a}$ will always lead to positive effects in all life cycle stages and the total population numbers, but that small values of $\mu_{a}$ will always lead to negative effects on the larval (and hence) pupal stages. (There is even the theoretical possibility of mixed results in which adults and total population numbers are positively effected while larval and pupal numbers are negatively effected.)

We have shown that the periodic LPA model (4) theoretically can predict positive effects of environmental periodicity under certain circumstances. This prediction depends upon model parameter constraints as described in Theorem 9. Are these constraints biologically realistic? That is, can the inequality (22) hold for known estimates of the model parameters for flour beetle cultures?

Table 1 contains five sets of parameter estimates for the LPA model taken from the literature. Parameter set 1 is obtained from Jillson's actual experimental data ${ }^{1}$. All of these parameter sets satisfy the inequality (22). Therefore, for these parameter values the periodic LPA model (4) predicts, at least for large values of $b$, a positive effect on total population numbers due to small amplitude environmental periodicities. While Theorem 9 applies only asymptotically as $b \rightarrow+\infty$, we see from Table 1 that the model predicted positive effect can in fact occur for relatively small values of $b$ (i.e. for $b>b_{\alpha}$ in Table 1);

\footnotetext{
${ }^{1}$ Data provided by Professor R. F. Costantino and statistical parameter estimates provided by Professor Brian Dennis.
} 
in particular, see parameter sets 1,2, and 4. Fig. 2 provides a graphical illustration of this fact for the Jillson parameter set 1 . In parameter sets 3 and 5 , however, the predicted positive effect is not seen prior to the destabilization of the constant environment equilibria.

Theorem 9 implies that a positive effect can be the result of environmental periodicity of small amplitude $\alpha$ in the sense that small amplitude 2-cycle solutions near the equilibrium solution of the autonomous model have increased averages for sufficiently large values of $b$. This result is independent of the stability properties of the unperturbed equilibrium or the 2-cycle solutions of the periodically perturbed system (which may be stable or unstable). The equilibrium of the unperturbed LPA model generally losses stability as $b$ increases $[14,16,28]$. Our analytical results do not address the question of whether this loss of stability occurs before or after the onset of the positive effect (i.e. for $b$ less than or greater than the quantity $b_{\alpha}$ in Table 1). This remains an open general question. In Table 1 the "cross over" from negative to positive effects as $b$ increases through $b_{\alpha}$ occurs before the equilibria lose stability for parameter sets 1,2 , and 4 , but not for parameter sets 3 and 5 .

Our results show that one particular model, designed specifically for the dynamics of a particular animal (flour beetles), predicts a positive effect due to periodic habitat fluctuations for realistic parameter values. These mathematical results provide an explanation of the increased biomass observed by Jillson [40] by means of a specific biological mechanism, namely the effect of periodic fluctuations of flour volume on cannibalism rates. The Jillson flour beetle experiment is studied in more detail using the periodic LPA model in [16]. These theoretical and experimental results together provide the first clear demonstration that increased population biomass is a possible consequence of environmental fluctuations. Such a possibility raises many other interesting biological and mathematical questions. For example, what role does such a possibility play in adaptation strategies for a species in a periodic environment, or how might a resource manager take advantage of such a possibility in order to increase biomass production without having to increase (the average amount) of environmental resources? Finally, how ubiquitous are positive effects of periodic forcing in mathematical models of population dynamics? This mathematical question is addressed for scalar maps and discrete systems of a particular form in [38].

Acknowledgements. The authors thank R. F. Costantino (Department of Biological Sciences, University of Rhode Island), Brian Dennis (Department of Wildlife and Forestry and Department of Statistics, University of Idaho), and Robert Desharnais (Department of Biology, California State University at Los Angeles) for their helpful comments and suggestions.

\section{References}

1. Abrams, P., 1984. Variability in resource consumption rates and the coexistence of competing species, Theor. Pop. Biol. 25: 106-124 
2. Bardi, M. and A. Schiaffino, 1982. Asymptotic behaviour of positive solutions of periodic delay logistic equations, J. Math. Biol. 14: 95-100

3. Bardi, M., 1983. An Equation of growth of a single species with realistic dependence on crowding and seasonal factors, J. Math. Biol. 17: 33-43

4. Boyce, M. S. and D. J. Daley, 1980. Population tracking of fluctuating environments and natural selection for tracking ability, Amer. Nat. 115: 480-491

5. Chesson, P. L., 1982. The stabilizing effect of a random environment, J. Math. Biol. 15: $1-36$

6. Chesson, P. L., 1986. Environmental variation and the coexistence of species. Community Ecology, J. Diamond and T. J. Case (Eds.), Harper and Row, New York, pp. $240-256$

7. Chesson, P. L., 1991. Stochastic population models, appearing in Ecological Heterogeneity, J. Kolasa and S. Pickett (eds.), Springer, New York, pp. 123-143

8. Chesson, P. L. and T. J. Case, 1986. Nonequilibrium community theories: chance, variability, history, and coexistence, Community Ecology, J. Diamond and T. J. Case (Eds.), Harper and Row, New York, pp. 229-239

9. Chesson, P. L. and S. Ellner, 1989. Invasibility and stochastic boundedness in monotonic competition models, J. Math. Biol. 27: 117-138

10. Chesson, P. L. and R. R. Warner, 1981. Environmental variability promotes coexistence in lottery competitive systems, Am. Nat. 117: 923-943

11. Cohen, D. S. and S. Rosenblat, 1982. A delay logistic equation with variable growth rate, SIAM J. Appl. Math. 42: 608-624

12. Coleman, B. D., 1979. Nonautonomous logistic equations as models of the adjustment of populations to environmental change, Math. Biosci. 45: 159-173

13. Costantino, R. F. and R. A. Desharnais, 1991. Population Dynamics and the Tribolium Model: Genetics and Demography, Monographs on Theoretical and Applied Genetics 13, Springer, Berlin

14. Costantino, R. F., J. M. Cushing, B. Dennis and R. A. Desharnais, 1995. Experimentally induced transitions in the dynamic behaviour of insect populations, Nature 375 : 227-230

15. Costantino, R. F., R. A. Desharnais, J. M. Cushing and B. Dennis, 1997. Chaotic dynamics in an insect population, Science 275: 389-391

16. Costantino, R. F., J. M., Cushing, B. Dennis, R. A. Desharnais, S. M. Henson, 1997. Resonant population cycles in alternating habitats, to appear in Bull. Math. Biol.

17. Cushing, J. M., 1977. Periodic time-dependent predator-prey systems, SIAM J. Appl. Math. 32: $82-95$

18. Cushing, J. M., 1980. Two species competition in a periodic environment, J. Math. Biol. 10: $385-400$

19. Cushing, J. M., 1982. Periodic Kolmogorov systems, SIAM J. Math. Anal. 13: 811-827

20. Cushing, J. M., 1983. Periodic Lotka-Volterra systems and time sharing of ecological niches, Lecture Notes in Biomathematics 52, Springer, Berlin. pp. 342-348

21. Cushing, J. M., 1984. Periodic two-predator, one-prey interactions and the time sharing of a resource niche, SIAM J. Appl. Math. 44: 392-410

22. Cushing, J. M., 1987. Oscillatory population growth in periodic environments, Theor. Pop. Biol. 30: 289-308

23. Cushing, J. M., 1995. Systems of difference equations and structured population dynamics, Proceedings of the First International Conference on Difference Equations, Saber N. Elaydi, John R. Graef, Gerry Ladas, Allan C. Peterson (Eds), Gordon and Breach Publishers, US, 1995, pp. 123-132

24. Cushing, J. M. and Zhou Yicang, 1994. The net reproductive value and stability in matrix population models, Nat. Res. Model, 8(4) : 297-333

25. Cushing, J. M., B. Dennis, R. A. Desharnais and R. F. Costantino, 1996. An interdisciplinary approach to understanding nonlinear ecological dynamics, Ecological Modelling 92: 111-119

26. de Mottini, P. and A. Schiaffino, 1979. On logistic equations with time periodic coefficients, I.A.C. Mauro Picone, Series III, No. 192, Rome

27. de Mottini, P. and A. Schiaffino, 1981. Competition systems with periodic coefficients: A geometric approach, J. Math. Biol. 11: 319-335 
28. Dennis, B., R. A. Desharnais, J. M. Cushing and R. F. Costantino, 1995. Nonlinear demographic dynamics: Mathematical models, statistical methods, and biological experiments, Ecol. Monogr. 65(3): 261-281

29. Dennis, B., R. A. Desharnais, J. M. Cushing and R. F. Costantino, 1997. Transitions in population dynamics: equilibria to periodic cycles to aperiodic cycles, to appear in $\mathrm{J}$. Animal Ecology

30. Ellner, S., 1987. Alternate plant life history strategies and coexistence in randomly varying environments, Vegetatio 69: 199-208

31. Ellner, S., 1989. Convergence to stationary distributions in two species competition models, J. Math. Biol. 27: 451-462

32. Gantmacher, F. R., 1959. The Theory of Matrices, Vol. II. Chelsea Publishing Company, New York

33. Guckenheimer, J. and P. Holmes, 1983. Nonlinear Oscillations, Dynamical Systems, and Bifurcations of Vector Fields, Springer-Verlag, New York, p. 18

34. Hale, J. K., 1988. Asymptotic behavior of dissipative systems, Math. Surveys Monogr. 25, Amer. Math. Soc., Providence. pp. 13,19

35. Hale, J. K. and A. S. Somolinos, 1983. Competition for fluctuating nutrient, J. Math. Biol. 18: $255-280$

36. Hsu, S. B., 1980. A competition model for a seasonally fluctuating nutrient, J. Math. Biol. 9: 115-132

37. Henson, S. M., 1996. Existence and stability of nontrivial periodic solutions of periodically forced discrete dynamical systems, J. Difference Eqn. and Appl. 2: 1137-1142

38. Henson, S. M., 1996. The effect of periodicity in maps, submitted

39. Hofbauer, J. and J. W. -H. So, 1989. Uniform Persistence and repellors for maps, Proc. Amer. Math. Soc. 107(4): 1137-1142

40. Jillson, D., 1980. Insect populations respond to fluctuating environments, Nature $\mathbf{2 8 8}$ : 699-700

41. Kuang, Y. and J. M. Cushing, 1995. Global stability in a nonlinear difference-delay equation model of flour beetle population growth, J. Difference Eqn. and Appl. 2: 31-37

42. Loreau, M., 1989. Coexistence of temporally segregated competitors in a cyclic environment, Theor. Pop. Biol. 36: 181-201

43. May, R. M., 1973. Stability and complexity in model ecosystems, Princeton University Press, p. 123

44. May, R. M. (Ed.) 1976. Theoretical Ecology. W. B. Saunders, p. 23

45. Murray, J. D., 1989. Mathematical Biology, Vol. 19, Biomathematics, Springer-Verlag, Berlin, p. 702

46. Namba, T., 1984. Competitive co-existence in a seasonally fluctuating environment, J. Theor. Biol. 111: $369-386$

47. Namba, T., 1986. Bifurcation phenomena appearing in the Lotka-Volterra competition equations: a numerical study, Math. Biosci. 81: 191-212

48. Nisbet, R. M. and W. S. C. Gurney, 1976. Population dynamics in a periodically varying environment, J. Theor. Biol. 56: 459-475

49. Rosenblat, S., 1980. Population models in a periodically fluctuating environment, J. Math. Biol. 9: 23-36

50. Sanchez, D. A., 1982. Periodic environments, harvesting, and a Riccati equation, Nonlinear Phenomena in Mathematical Sciences, V. Laksmikantham (Ed.), Academic Press, New York

51. Smith, H. L., 1981. Competitive coexistence in an oscillating chemostat, SIAM J. Appl. Math. 40: 498-522

52. Smith, H. L. and P. Waltman, The Theory of the Chemostat, Cambridge Studies in Mathematical Biology, Cambridge University Press, Cambridge, Chapter 7

53. Sonneveld, P. and J. van Dan, 1979. On a conjecture about the periodic solutions of the logistic equations, J. Math. Biol. 8: 285-289

54. Stanley, J., 1932. A mathematical theory of the growth of populations of the flour beetle, Tribolium confusum, Duv. Can. J. Res. 6: 632-671

55. Turelli, M., 1981. Niche overlap and invasion of competitors in random environments I. Models without demographic stochasticity, Theor. Pop. Biol. 20: 1-56 